\title{
0 papel da oxigenação hiperbárica na estrutura do fígado e baço após ligadura das veias hepáticas: estudo em ratos
}

\author{
The role of hyperbaric oxygenation in the liver and spleen structure \\ after hepatic vein ligation: study in rats
}

\author{
Ricardo C osta-Val ${ }^{1}$, Tarcizo Afonso $\mathrm{N}_{\text {unes }}{ }^{2}$, Roberto Carlos de 0 liveira e Silva ${ }^{3}$
}

\begin{abstract}
Resumo
O bjetivo: Avaliação morfológica do fígado e baço de ratos submetidos à oxigenoterapia hiperbárica após a ligadura das veias hepáticas.

M étodo: Foram utilizados 30 animais machos adultos da espécie $\mathrm{H}$ oltzman, distribuídos aleatoriamente em dois grupos de 15 animais cada, assim designados: grupo 1 - ligadura das veias hepáticas; grupo 2 - ligadura das veias hepáticas associada à oxigenoterapia hiperbárica. Todos os animais foram submetidos à anestesia geral por meio de solução contendo cloridrato de cetamina $(40 \mathrm{mg} / \mathrm{ml})$ e cloridrato de meperidina ( $10 \mathrm{mg} / \mathrm{ml}$ ) na dose de $50 \mathrm{mg} / \mathrm{kg} /$ peso, laparotomia mediana eligadura das veias hepáticas. A oxigenoterapia hiperbárica foi aplicada nos animais do grupo 2, a partir da oitava hora do pós-operatório, por 120 minutos, sendo 90 minutos sob pressão de 2,5 atmosferase 15 minutos no início e final da terapêutica, para promover a compressão e descompressão gradativa no período de 20 dias consecutivos. No $21^{\circ}$ dia de pós-operatório, os animais foram mortos por inalação de éter e submetidos à laparotomia e extirpação dos fígados e baços para exame histológico. Foram comparados os resultados da histologia hepática e esplênica aplicando-se o teste exato de Fisher, considerando-se a diferença significante de $\mathrm{P}<0,05$.

Resultados: 0 s exames histológicos dos fígados e baços dos animais dos grupos 1 e 2 mostraram as seguintes alterações: presença de trombose nas veias hepática, porta e centro-lobular em cinco (33,3\%) animais do grupo 1 e ausência no grupo 2; presença de necrose dos hepatócitos caracterizada como acentuada em sete animais $(46,7 \%)$ e leve em oito $(53,3 \%)$ animais do grupo 1 , enquanto que, em todos os animais do grupo 2, esta alteração foi caracterizada como leve; presença de células de Kupffer muito proeminentes e hipertrofiadas em 14 $(93,3 \%$ ) animais do grupo 1 e pouco proeminentes ehipertrofiadas em todos os animais do grupo 2; congestão da pol pa vermel ha considerada acentuada em seis (40\%) e moderada em nove (60\%) animais do grupo 1 e em todos os animais do grupo 2; hemossiderose moderada ou
\end{abstract}

\begin{abstract}
O bjective: Liver and spleen morphologic evaluation of rats submitted to hyperbaric oxygen therapy after hepatic vein ligation.

M ethod: Thirty $\mathrm{H}$ oltzman adult male rats were used, distributed into two groups of 15 animals: group 1 - hepatic vein ligation; group 2 - hepatic vein ligation associated with hyperbaric oxygen therapy. All animal s received general anesthesia by a solution composed of ketamine chloride $(40 \mathrm{mg} / \mathrm{ml}$ ) and meperidine chloride $(10 \mathrm{mg} / \mathrm{ml})$ in a dose of $50 / \mathrm{mg} /$ weight, and were submitted to median laparotomy and hepatic vein ligation. G roup 2 animals were submitted to hyperbaric oxygen therapy, 8 hours after the operation, 90 minutes at 2.5 atmosphere pressure and 15 minutes at the onset and end of the therapy, in a total of 120 minutes, in order to promote the gradual compression and decompression in 20 consecutive days. 0 n the $21^{\text {st }}$ preoperative day, the animals were sacrificed by ether inhalation and submitted to laparotomy and stripping of liver and spleen for histological study. The results of the histological study of livers and spleens were compared using Fisher's exact test. Statistical ly significant difference was considered when $P<0.05$.

Results: The histological studies made in the livers and spleens of animals from both groups showed the following alterations: presence of thrombosis of hepatic, portal and central lobular veins in five (33.3\%) group 1 animals and absence in group 2 animals; very extensive necrosis of liver cells in seven $(46.7 \%)$ group 1 animals, and light in eight $(53.3 \%)$, whereas for all group 2 animals such alteration was considered light; Kupffer cells developed and hypertrophied in 14 (93.3\%) group 1 animals and slightly developed and hypertrophied in all group 2 animals; high congestion of the spleen purple in six $(40 \%)$ and moderate in nine (60\%) group 1 animals, whereas all group 2 animals had moderate congestion; moderate or severe hemosiderosis in 14 (93.3\%) group 1 animals and mild hemosiderosis in all group 2 animals. The statistical
\end{abstract}

1. Professor, Universidade Vale do Rio Verde (UNINCOR), Belo Horizonte, MG. Doutor em Cirurgia, Programa de Pós-Graduação em Cirurgia, Faculdade de Medicina da Universidade Federal de Minas Gerais (UFMG), Belo Horizonte, MG. Membro titular da Sociedade Brasileira de Angiologia e de Cirurgia Vascular.

2. Doutor. Professor adjunto, Departamento de Cirurgia, Faculdade de Medicina da UFMG, Belo Horizonte, MG. Coordenador, Programa de Pós-Graduação em Cirurgia, UFMG, Belo Horizonte, MG.

3. Doutor em Cirurgia, Programa de Pós-Graduação em Cirurgia, Faculdade de Medicina, UFMG, Belo Horizonte, MG. Professor substituto, Departamento de Cirurgia, Faculdade de Medicina, UFMG, Belo Horizonte, MG.

Artigo submetido em 13.12.05, aceito em 10.02.06. 
acentuada em 14 (93,3\%) animais do grupo 1 e leve em todos os animais do grupo 2. As análises estatísticas realizadas entre os dois grupos mostraram diferenças significativas em todas a variáveis estudadas $(P<0,05)$.

C onclusões: A oxigenoterapia hiperbárica em ratos submetidos à ligadura das veias hepáticas atenuou os efeitos del etérios e precoces sobre o fígado e o baço, analisada pela histologia destes órgãos.

Palavras-chave: 0 xigenoterapia hiperbárica, ratos, histologia do fígado e baço. analyses performed between both groups showed significant differences $(P<0.05)$ for all variables.

Conclusions: The hyperbaric oxygen therapy applied in rats submitted to hepatic vein ligation mitigated its early deleterious effects on the liver and spleen, which was confirmed by the histological study. spleen.

Key words: H yperbaric oxygen, rats, histological study of liver and
A síndrome dehipertensão porta, caracterizada por hepatoesplenomegalia, circulação colateral hepatofuga e ascite, é uma doença freqüente e comum na prática médica, mas possui variada etiologia nas diferentes regiões do mundo, até mesmo dentro de um mesmo país. Suas principais complicações são a hemorragia digestiva alta e a insuficiência hepática. Seus fatores etiológicos mais comuns são a cirrose e a esquistossomose. Apesar de mais raras, as obstruções venosas podem ser graves, sobretudo quando ocorrem nas veias hepáticas ${ }^{1-4}$.

Jáa síndromede Budd-C hiari decorreda obstrução primária ou secundária da drenagem venosa do fígado e também possui várias etiologias. Suas manifestações clínicas podem ocorrer de maneira aguda ou crônica, mas as alterações nas provas das funções hepáticas são mais evidentes nos eventos agudos. Seu diagnóstico definitivo geralmente é feito por métodos de imagem associados à histologia do fígado. N o tratamento desta síndrome, podem ser empregados agentes farmacológicos, derivações cirúrgicas porta-sistêmicas (intra-hepáticastransjugulares, porta-cavaemesentérico-cava) e/ ou dispositivosendovasculares, maso transplantehepático ortotópico costuma ser o tratamento definitivo nos casos mais graves ou de difícil controle por outras medidas. A pesar disso, esse procedimento écomplexo e não pode ser empregado em todos os pacientes, devido ao quadro clínico, bem como pela falta de órgãos e serviços especializados, o que, geralmente, resulta em longo tempo de espera. $D$ evido a isso, muitospacientes com insuficiência hepática não sobrevivem até o momento do transplante ${ }^{5-8}$.

É relevante, portanto, pesquisar novos métodos terapêuticos capazes de amenizar ou combater os efeitos da obstrução das veias hepáticas sobre o fígado, oferecendo aos doentes a possibilidade demelhoria das condições clínicas enquanto aguardam 0 tratamento definitivo.
A oxigenoterapia hiperbárica tem se mostrado capaz de melhorar a evolução de animais e seres humanos com diversas afecções, nas quais existe, em suas fisiopatologias, a tríade: hipóxia, isquemia e reperfu são ${ }^{9-14}$. Sabe-se que a congestão venosa grave podeinduzir isquemia celular, principalmente quando ocorre de forma súbita15,16. Sua aplicabilidade e indicação da oxigenoterapia hiperbárica estão asseguradas cientificamente para várias doenças, mas existe vasto campo da área médica no qual sua eficácia é desconhecida, sendo necessários adicionais estudos experimentais e clínicos ${ }^{17-20}$.

Seguindo essa linha de raciocínio, realizou-se esta pesquisa, cuja hipótese inicial era a de avaliar os efeitos da oxigenoterapia hiperbárica em ratos submetidos à congestão hepática por meio da ligadura das veias hepáticas.

\section{M aterial e método}

Este estudo seguiu os princípios éticos na experimentação animal descritos pelo C olégio B rasileiro de Experimentação Animal (COBEA) e foi aprovado pela $C$ âmara do $D$ epartamento de C irurgia da Faculdade de $M$ edicina da U niversidade Federal de $M$ inas Gerais. Para a realização do experimento, foram empregados 30 ratos machos $\mathrm{H}$ oltzman, animaisque possuem características biológicas semelhantes aos ratos $\mathrm{W}$ istar. Os ratos permaneceram em gaiolas apropriadas, expostos à luz em ciclos regulares de 12 horas, e receberam ração industrial ( $N$ uvilab $\mathrm{CR}_{1}{ }^{\circledR}$, Laboratório $\mathrm{N}$ uvital) e água ad libidum em todas as fases do estudo.

Os animais foram distribuídos, aleatoriamente e por sorteio, em dois grupos:

G rupo 1 - ligadura das veias hepáticas: 15 animais submetidos à congestão hepática induzida por meio da ligadura das veias hepáticas; 
G rupo 2 - ligadura das veias hepáticas associada à oxigenoterapia hiperbárica: 15 animais submetidos à congestão hepática induzida por meio da ligadura das veias hepáticas e à oxigenoterapia hiperbárica.

\section{Procedimento cirúrgico}

O s ratos foram submetidos a anestesia geral mediante injeção no músculo glúteo superficial direito da solução de $8 \mathrm{ml}$ de cloridrato de cetamina $(40 \mathrm{mg} / \mathrm{ml}$ ) e $2 \mathrm{ml}$ decloridrato demeperidina $(10 \mathrm{mg} / \mathrm{ml})$, na dose de $50 \mathrm{mg} / \mathrm{kg} /$ peso, referente ao cloridrato de cetamina. Realizou-se a tonsura dos pêlos do tórax e abdômen ea anti-sepsia com solução al coólica de polivinil pirrolidona-iodo a $1,0 \%$.

A operação iniciou-se pela laparotomia mediana, que se estendeu a partir do terço inferior do apêndice xifóide até o púbis, seguida de exploração da cavidade abdominal, dissecção delicada do ligamento redondo do fígado e identificação das veias hepáticas. T odas as veiashepáticasforam dissecadas eligadas, em conjunto, com fio de seda trançada e siliconizada 4-0, seguindo a mesma padronização da técnica operatória em todos animais operados. A seguir, foram feitas a revisão da hemostasia e a síntese da cavidade abdominal em duas camadas de sutura contínua com fio de nylon 3-0.

\section{Pós-operatório}

Após os procedimentos cirúrgicos, os animais foram alojados em gaiolas individuais até a recuperação anestésica, sendo agrupados, posteriormente, em gaiolas coletivas e examinados em intervalos regulares de aproximadamente quatro horas nas primeiras 48 horas e de oito horas após os 2 primeiros dias, até o término do experimento, sendo excluídos do estudo todos os animais que morreram nas primeiras quatro horas ou que não apresentaram boa recuperação cirúrgico-anestésica nas primeiras oito horas de pós-operatório.

\section{O xigenoterapia hiperbárica}

Empregou-se câmara hiperbárica tipo monopaciente (modelo BLKS 303 M K, M oscou), aprovada para uso no Brasil pela A gência $\mathrm{N}$ acional de V igilância Sanitária (AN VISA). A oxigenoterapia hiperbárica nos animais do grupo 2 foi aplicada em uma sessão diária durante 20 dias consecutivos, em intervalos regulares de 24 horas, que se iniciou na oitava hora após o término das operações nos animais deste grupo. Foi administrado oxigênio a $100 \%$, com duração total de 120 minutos cada sessão, sendo 90 minutos sob pressão de 2,5 atmosferas e os 15 minutos iniciais efinais para compressão e descompressão gradativas, respectivamente.

\section{Morte dos animais e retirada dos órgãos}

O s fígados e baços dos animais dos dois grupos foram extirpados e fixados em formol a 10\% para estudo microscópico, sendo este procedimento real izado logo após a constatação do óbito, a fim de se evitar as alterações degenerativas dos órgãos nos animais que morreram e que não foram excluídos, conforme citado anteriormente. Já os animais que permaneceram vivos foram submetidos à eutanásia por inalação de éter, para a extirpação do fígado e baço de acordo com a padronização estabelecida:

Grupo 1 - 21ำ dia de pós-operatório;

Grupo $2-21^{\circ}$ dia de pós-operatório, após as 20 sessões de oxigenoterapia hiperbárica.

\section{Histologia}

Fígados e baços foram processad os em blocos de parafina, submetidos a cortes histológicos longitudinais em micrótomo calibrado para 5 micra e corados com hematoxilina e eosina, para estudo à microscopia de luz.

\section{Variávés estudadas}

Foram pesquisadas alterações histológicas dos fígados e baços entre os animais dos dois grupos, e estasforam comparadas utilizando-se o testeexato de Fisher, considerando-se um nível de significância de $5 \%(P<0,05)$.

\section{Resultados}

\section{H istologia hepática dos animais dos grupos 1 e 2}

A trombose nas veias hepática, porta e centrolobular foi diagnosticada em cinco $(33,3 \%)$ dos 15 animais do grupo 1 e em nenhum dos animais do grupo 2. Esse resultado foi significativo $(P<0,05)$ (T abelas 1 e 2 e Figura 1).

O bservou-se necrose dos hepatócitos de grau acentuado em sete $(46,7 \%)$ e leve em oito $(53,3 \%)$ dos 15 animais do grupo 1. Em todos os animais do grupo 2, essa al teração histopatológica foi consideradaleve, reve- 
lando diferença significativa entre os dois grupos $(\mathrm{P}<0,05)$ (T abela 3).

Já a presença de células de K upffer muito proeminentes e hipertrofiadas foi observada em 14 $(93,3 \%)$ dos animais do grupo 1 . Em todos do grupo 2, o diagnóstico foi de células de Kupfer pouco proeminentes e hipertrofiadas. Esse resultado mostrou diferença significativa entre os dois grupos $(P<0,05)$ (T abela 4).

T abela 1 - Comparação dos animais dos grupos 1 e 2 quanto à presença de trombose nas veias hepáticas, porta e centro-lobular

\begin{tabular}{lcccc}
\hline Trombose nas veias hepática, & \multicolumn{2}{c}{ G rupo 1 } & \multicolumn{2}{c}{ G rupo 2 } \\
porta e centro-lobular & n & \% & n & \% \\
\hline Ausente & 10 & 66,7 & 15 & 100,0 \\
Presente & 5 & 33,3 & 0 & 0,0 \\
Total & 15 & 100,0 & 15 & 100,0 \\
\hline
\end{tabular}

$P \leq 0,042$ ( $P$ se refere ao teste exato de Fisher).
Tabela 2 - Comparação dos animais dos grupos 1 e 2 quanto à presença de necrose de hepatócitos

\begin{tabular}{lcccc}
\hline Necrose de hepatócitos & \multicolumn{2}{c}{ Grupo 1 } & \multicolumn{2}{c}{ Grupo 2 } \\
& n & $\%$ & n & $\%$ \\
\hline Leve & 8 & 55,3 & 15 & 100,0 \\
Acentuada & 7 & 46,7 & 0 & 0,0 \\
Total & 15 & 100,0 & 15 & 100,0 \\
\hline
\end{tabular}

$P=0,006$ ( $P$ se refere ao teste exato de Fisher)

Tabela 3 - Comparação dos animais dos grupos 1 e 2 quanto à presença de células de K upffer proeminentes e hipertrofiadas

\begin{tabular}{lcccc}
\hline Células de Kupffer proemi- & \multicolumn{2}{c}{ G rupo $\mathbf{1}$} & \multicolumn{2}{c}{ G rupo 2 } \\
nentes e hipertrofiadas & n & $\%$ & n & $\%$ \\
\hline Pouco & 1 & 6,7 & 15 & 100,0 \\
M uito & 14 & 93,3 & 0 & 0,0 \\
Total & 15 & 100,0 & 15 & 100,0 \\
\hline
\end{tabular}

$P<0,001$ ( $P$ se refere ao teste exato de Fisher).
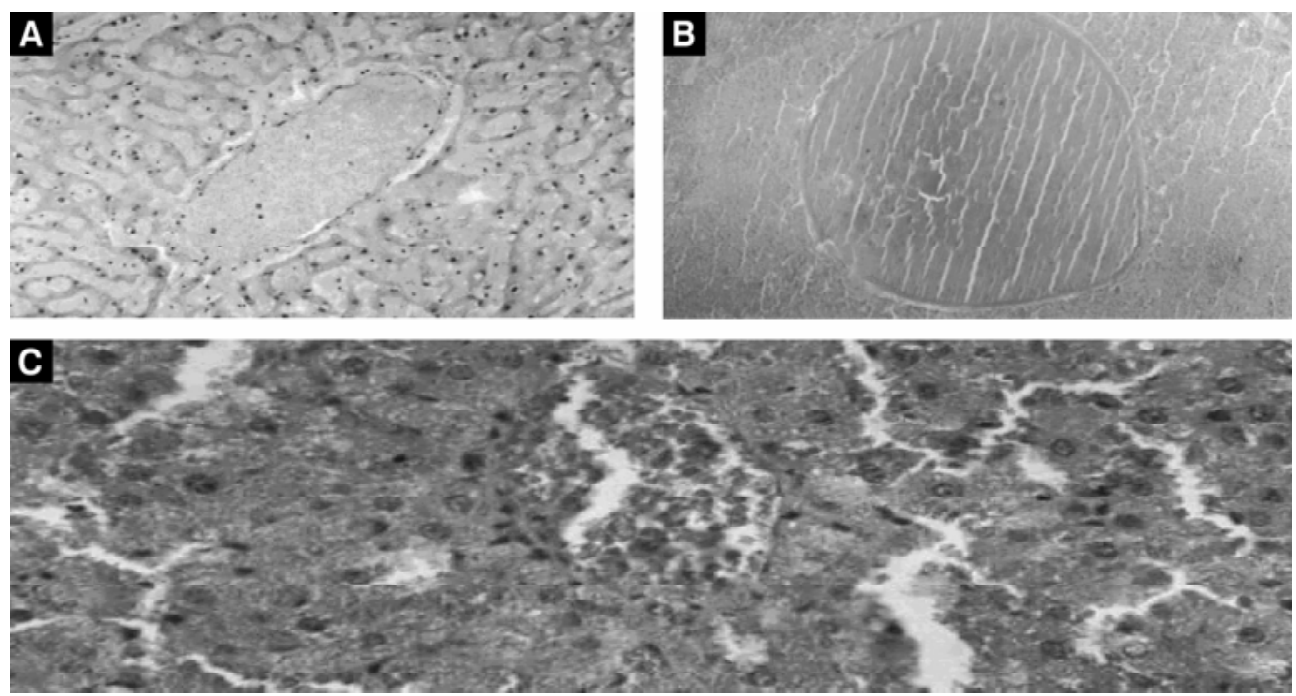

Figura 1 - Estudo histológico, por microscopiaótica, dosfígados deanimais do grupo 1 (A eB) egrupo 2 (C), nos quaissediagnosticaram: trombose da veia hepática $(\mathbf{A})$; trombose centro-lobular com necroseassociada (B); estasecentro-lobular associada à dilatação sinusoidal, além de congestão importante(C). Coloração pela hematoxilina-eosina. Aumentos de 200X (A e B) e 400X (C) 


\section{H istologia esplênica dosanimaispertencentesaosgrupos 1 e 2}

No grupo 1, foi evidenciada congestão acentuada dapol pavermelha em seis $(40,0 \%)$ animais, e, em todos do grupo 2, a congestão da polpa vermelha foi considerada moderada, mostrando diferença significativa entre os dois grupos $(P<0,05)$ (T abela 5 e Figura 2$)$.

D os 15 animais do grupo 1, 14 (93,3\%) apresentaram hemossiderose moderada ou acentuada; no grupo 2, essa alteração foi diagnosticada como leve, sendo a diferença significativa $(P<0,05)$ (Figura 2).

T abela 4 - Comparação dosanimaisdosgrupos1 e2 quanto à presença de congestão da pol pa vermelha

\begin{tabular}{lcccc}
\hline $\begin{array}{l}\text { Congestão da } \\
\text { polpa vermelha }\end{array}$ & \multicolumn{2}{c}{ G rupo 1 } & \multicolumn{2}{c}{ Grupo 2 } \\
& n & $\%$ & n & $\%$ \\
\hline M oderada & 9 & 60,0 & 15 & 100,0 \\
Acentuada & 6 & 40,0 & 0 & 0,0 \\
Total & 15 & 100,0 & 15 & 100,0 \\
\hline$P=0,017$ (P se refere ao teste exato de Fisher).
\end{tabular}

\section{D iscussão}

A oxigenoterapia hiperbárica tem sido objeto de estudos, principalmente em nível experimental, por questões éticas, e suas propriedades biológicas, bem como mecanismos de atuação, começam a ser melhores compreendidos, apesar deexistirem ainda grandeslacunasa serem preenchidas. Apesar determosusado câmara hiperbárica do tipo monopaciente neste trabalho, existem variações da mesma exclusivas para pesquisas em animais ${ }^{21}$. Entretanto, os achados histológicos observados neste experimento demonstram que o modelo

Tabela 5 - Comparação dos animais dos grupos 1 e 2 quanto à hemossiderose

\begin{tabular}{lcccc}
\hline H emossiderose & \multicolumn{2}{c}{ Grupo 1 } & \multicolumn{2}{c}{ Grupo 2 } \\
& n & $\%$ & n & $\%$ \\
\hline Leve & 1 & 6,7 & 15 & 100,0 \\
M oderada/acentuada & 14 & 93,3 & 0 & 0,0 \\
Total & 15 & 100,0 & 15 & 100,0 \\
\hline$P<0,001(P$ a
\end{tabular}

$P<0,001$ ( $P$ se refere ao teste exato de Fisher).
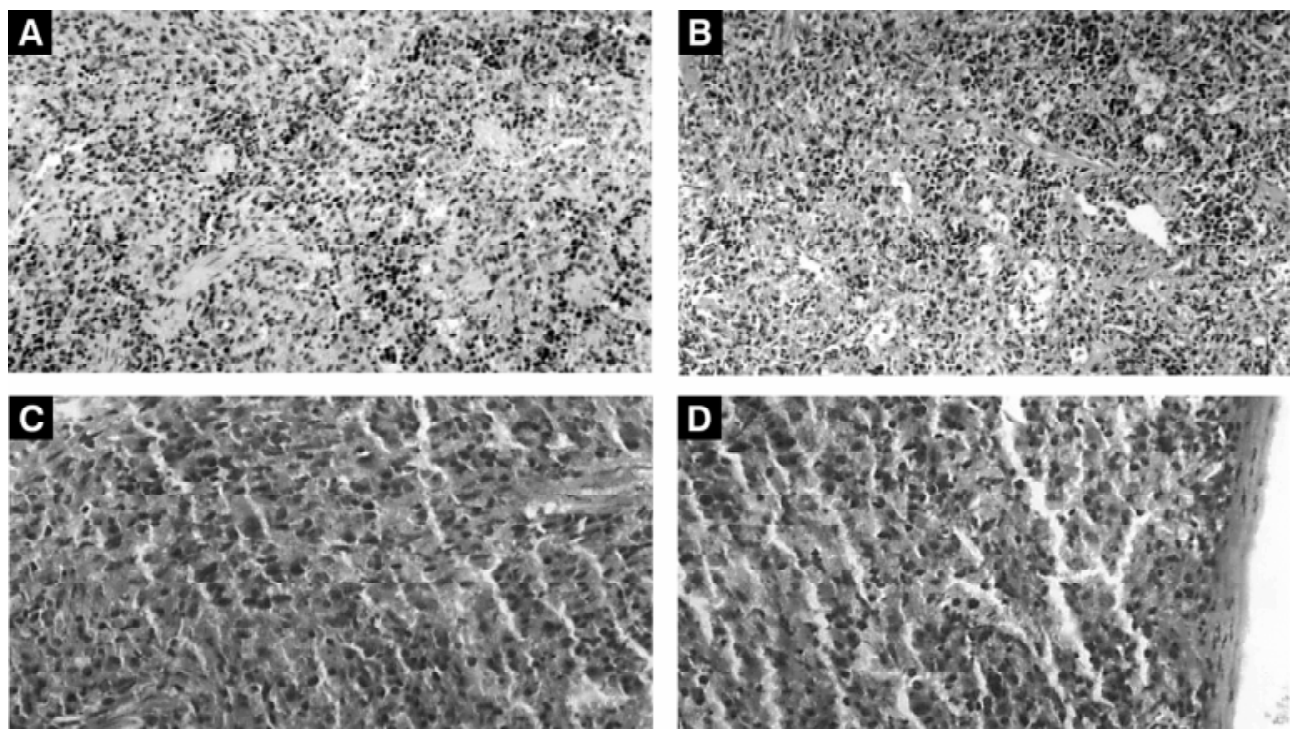

Figura 2 - Estudo histológico, por microscopia ótica, dos baços de animais do grupo 1 (A eB) e grupo 2 ( $\mathbf{C}$ e $\mathbf{D}$ ), nos quais se diagnosticaram estase esplênica associada à hemossiderinose moderada (A); estase sinusoidal de grau moderado da polpa vermelha (B); congestão importante da polpa vermelha (C); na região subcapsular esplênica, a presençade congestão moderada da polpa vermelha (D ). Coloração pela hematoxilina-eosina. Aumentos de 200X (A e B) e 400X (C e D) 
de estudo utilizado cumpriu a proposta de criar-se um estado hipertensivo venoso no sistema hepático e, portanto, compatível com a hipótese inicial.

As alterações histológi cas e os resultad os observados no grupo 2 são também compatíveis com dados da literatura, inclusive de pesquisas nacionais, realizadas em ratos, que mostraram a diminuição da agregação plaquetária e leucocitária e do edema secundário aos fenômenos de reperfusão e da hiporreatividade de macrófagos e das células de Kupffer hepáticas, fenômenos estes já descritos como conseqüências biológicas relacionadas à oxigenoterapia hiperbárica e à inibição do óxido nítrico. A atenuação das alterações histopatológicas encontradas no grupo 2 parecem relacionar-se à capacidade da oxigenoterapia hiperbárica de reduzir a resposta inflamatória sistêmica após condições clínicas graves e agir de maneira atenuante, o que também já foi descrito na literatura como uma propriedade da oxigenação hiperbárica22-27.

Estudos demonstraram ainda que o tratamento hiperbárico, imediatamente após eventos isquêmicos, diminui os fenômenos del etérios secundários à síndrome de reperfusão de forma sistêmica e não apenas local ${ }^{9-11}$. De acordo com dados da literatura, com apenas uma hora após o emprego da oxigenoterapia hiperbárica em ratos, foi detectada redução da agregação leucocitária no endotélio venoso, as paredesvenosas da musculatura esquelética apresentaram-se livres e bem definidas histologicamente dos fenômenos de reperfusão, além de ter ocorrido inibição da isquemia causada pela vasoconstrição microarteriolar, secundáriaaoseventosdereperfusão ${ }^{12,13}$. Portanto, o tempo de início da oxigenoterapia hiperbárica éimportante, uma vez que a hiperóxia al tera as vias bioquímicas responsáveis pela produção de radicaislivres em favor de produtosmenostóxicosaostecidos, al ém dereduzir a ativação endotelial, o que diminui a atração dos neutrófilos ${ }^{11}$. Esta variável foi levada em consideração neste estudo, por isso iniciamos a administração do oxigênio hiperbárico já nas primeiras horas de pós-operatório. 0 tempo de oito horas foi definido com o intuito de se esperar a recuperação pós-operatória dos animais, minimizando, assim, possíveis fatores de confusão. 0 utro mecanismo pelo qual a oxigenoterapia hiperbárica atua sobre os neutrófilos se dá por meio da seqüestração preferencial dos neutrófilos primários em órgãos mais sensíveis à hiperóxia, tais como os pulmões, o que inibe a liberação dessas células na corrente sangüínea-11.
Jáa estase venosa graveal tera o metabolismo celular eosmecanismos de coagulação, sendo as células vermeIhas e brancas da microcirculação local um dos principais agentes celulares diretamente responsáveis pela produção de radicais livres ${ }^{23}$. A oferta de $\mathrm{O}_{2}$ em altas doses torna-se benéfica nesta situação, pois é capaz de diminuir a produção da enzima superóxido dismutase, originada durante eventos isquêmicos e que está envolvida na produção de outros radicaislivresetóxicos para a biologia celular ${ }^{22-24}$. É importante salientar também que, por meio de estudos experimentais, concluiu-se que osinibidores do óxido nítrico atenuaram a hipotensão e a hiporreatividade vascular, inclusive em afecções circulatórias, além de reduzirem a lesão hepática avaliada por dosagens bioquímicas $25,26,28$. N os fenômenos de isquemia e reperfusão, os genes celulares favorecem a condição inflamatória queinduz a ativação ea agregação leucocitária e sua migração transcapilar, gerando, com isso, um desequilíbrio do ciclo do óxido nítrico, o queacarreta aumento dos superóxidos. U ma das conseqüências finais desse processo é o desequilíbrio da resposta vasomotora e o aumento da agregação plaquetária, dos fenômenos trombóticos e da necrose muscular. G rande parte destes achadosforam prevenidos após a administração da L-arginina, substância sabidamente capaz de interferir na produção do óxido nítrico ${ }^{14}$.

Sabe-se que o óxido nítrico é uma substância sintetizada a partir de um átomo de nitrogênio terminal guanidino da L-arginina por um processo distinto de classes de enzimas, classificadas como constitutivas (endotelial e neuronal) e que induzem a formação de outra enzima denominada óxido nítrico sintetase. Estas enzimas constitutivas sintetizam e liberam o óxido nítrico dentro de segundos, em resposta a qualquer evento que cause a entrada extracelular súbita de cál cio ou, ainda, a partir da elevação do cálcio intracelular. Já as enzimas induzidas necessitam de al gum tempo para se expressarem e, a partir desse momento, produzem óxido nítrico até que ocorra a morte celular ou até que o substrato e os co-fatores para sua produção sejam degradados ${ }^{29,30}$. As expressões de genes responsáveis pela síntese do óxido nítrico foram encontradas em hepatócitos e nas células de Kupffer de ratos com hepatopatias induzidas, o que sugere importante papel desse elemento nas afecções hepáticas. M as, apesar da crescente evidência correlacionando o óxido nítrico com várias afecções, inclusive as hepatopatias, sua constatação torna-se difícil devido à sua meia vida, queé de poucos segundos ${ }^{28}$. 
O utros autores observaram que a hiperóxia, base fundamental da oxigenoterapia hiperbárica, é um dos agentes capazes de provocar a inibição da produção do óxido nítrico ${ }^{28}$. Existem diversas doenças correlacionadas àsalterações na capacidade dos vasos sangüín eosem produzir o óxido nítrico, denominadas disfunções endoteliais. Embora ainda não compreen didas completamente, essas disfunções endoteliais estão relacionadas a distúrbios da hemostasia edosfenômenos trombóticos, secundários aos diversos tipos de lesões celulares ${ }^{25}$. $M$ azariegos et al. descreveram a utilização da oxigenoterapia hiperbárica em seres humanos, particularmente em crianças, com quadro clínico grave de isquemia hepática, secundária à trombose da artéria hepática, ocorrida após o transplante hepático ortotópico. A análise do grupo de crianças submetidas à oxigenação hiperbárica eque foram comparadas com um grupo de crianças não submetidas a esta modalidade terapêutica - denominado grupo-controle - evidenciou diferença significativa em relação à diminuição de eventos hepáticos graves, como necroce, colangitee abscessos. H ouve ainda diferença significativa no tempo do retransplante hepático nas crianças submetidas à oxigenoterapiahiperbárica. Esteestudo possui grandeimportância, pois existe uma escassez de trabal hos citados nas fontes de dados, em língua inglesa, a respeito da oxigenoterapia hiperbárica perante afecções hepáticas ${ }^{31}$. 0 trabaIho aqui citado cumpriu com seu objetivo inicial, que foi avaliar um possível benefício da oxigenoterapia hiperbárica nas afecções hepáticas. Além depossibilitar uma possível linha de pesquisa, acreditamos que 0 maior entendimento da oxigenoterapia hiperbárica e do próprio óxido nítrico, descrito como um dos maioresachados da medicina no século XX, possibilitará que novas estratégias terapêuticas despontem no horizonte.

\section{C onclusão}

N este estudo, pôde-se concluir que a oxigenoterapiahiperbárica em ratossubmetidosà ligadura dasveias hepáticas atenuou os efeitos deletérios e precoces sobre o fígado e o baço dos animais, observados pela análise histológica.

\section{Referências}

1. Sherlock S. O sistema venoso porta e hipertensão porta. In: $D$ oenças do fígado edo sistemabiliar. R io deJ aneiro: $G$ uanabara Koogan; 1991. p. 109-58.
2. Johansen KH. Portal hypertension. In: Cronenwett JL, Rutherford RB. Decision making in vascular surgery. Philadelphia: W B Saunders; 2001. p. 306-10.

3. Bhasin DK, Malhi NJS. Variceal bleeding and portal hypertension: much to learn, much to explore. Endoscopy. 2002;34:119-28.

4. N unes TA, M iranda GH G. Q uando indicar o tratamento cirúrgico da hemorragia digestiva alta? In: Savassi-R ocha PR, Coelho LGV, Diniz MTC, Nunes TA. Tópicos em gastroenterologia. 13ㄹ ed. Rio de Janeiro: M ED SI ; 2003. p. 279-92.

5. V alla D C. H epatic vein thrombosis (Budd-C hiari syndrome). Semin Liver D is. 2002;22:5-14.

6. Janssen H L, Garcia-Pagan JC, Elias E, et al. Budd-Chiari syndrome: a review by an expert panel. J H epatol. 2003;38: 364-71.

7. $X u P Q, D$ ang $X W$. T reatment of membranous B udd-C hiari syndrome: analysis of 480 cases. $H$ epatobiliary Pancreat $D$ is Int. 2004;3:73-6.

8. M enon KV, Shah V, K amath PS. TheBudd-C hiari syndrome. N Engl J M ed. 2004;350:578-85.

9. N ylander $G$, Lewis D , N ordstom H , Larsson J. Reduction of postischemic edema with hyperbaric oxygen. Plast Reconstr Surg. 1985;76:596-603.

10. EdwardsRJ, Im M J, H oopesJE. Effects of hyperbaric oxygen preservation on rat limb replantation: a preliminary report. Ann Plast Surg. 1991;27:31-5.

11. Zamboni WA, Roth AC, Russell RC, Graham B, Suchy H, Kucan JO. Morphologic analysis of the microcirculation during reperfusion of ischemic skeletal muscle and the effect of hyperbaric oxygen. Plast Reconstr Surg. 1993;91:1110-23.

12. Zamboni W A.T hemicrocirculation and ischemia-reperfusion: basic mechanisms of hyperbaric oxygen. In: Kindall EP, W helan HT. H yperbaric medicine practice. Flagstaff: Best Publishing; 1999. p. 779-95.

13. Chen MF, Chen HM, U eng SW, Shyr MH. H yperbaric oxygen pretreatment attenuates reperfusion injury. Liver. 1998;18:110-6.

14. Chaudry IH, W ang $P$, Jarrar D. Ischemia and reperfusion injury. In: Rich N M, M attox KL, Hirshberg A. Vascular trauma. Philadelphia. Elsevier/Saunders; 2004. p. 73-84.

15. O rloff M J, D aily PO, G irard B. T reatment of Budd-C hiari syndrome due to inferior vena cava occlusion by combined portal and vena caval decompression. Am J Surg. 1992;163:137-42.

16. Ponce Gonzales JF, D ominguez Adame Lanuza E, M artin Zurita I, M orales M endez S. Portal hypertensive colopathy: histologic appearance of the colonic mucosa. H epatogastroenterology. 1998;45:40-3.

17. Grim PS, Gottlieb LJG, Boddie A, Batson E. H yperbaric oxygen therapy. JAM A. 1990;263:2216-20.

18. Costa-Val R, Silva RCO, N unes TA, Souza T KD P. O papel da oxigenoterapia hiperbárica na doença vascular periférica. J Vasc Br. 2003;2:177-82.

19. LimaEB, Bernardes CH A, M artins ACG, M arcondes CM . $O$ papel da oxigenoterapiahiperbáricano tratamento dagangrena gasosa clostridiana e da fasciite necrotizante. J Vasc Br. 2003;2:220-4.

20. M affei FH A. O xigenoterapia hiperbárica [editorial]. J Vasc Br. 2003;2:169. 
21. Lyra PereiraM L, T elesAP, PereiraN eto J . C âmarahiperbárica de acrílico para animais de pequeno porte. Acta Cir Bras. 2001;16:267-70.

22. Angel M F, Ramasastry SS, Swartz W M , N arayanan K, K uhns $D B, B$ asford RE, et al. The critical relationship between free radical sand degrees of ischemia: evidencefor tissueintolerance of marginal perfusion. Plast Reconstr Surg. 1988;81:233-9.

23. $H$ ammarlund $C$, Sundberg $T$. H yperbaric oxygen reduced size of chronic leg ulcers: a randomized double-blind study. Plast Reconstr Surg. 1994;93:829-33.

24. Hammarlund $C$. The physiologic effects of hyperbaric oxygenation. In: Kindall EP, W helan H T . H yperbaricmedicine practice. Flagstaff: Best Publishing; 1999. p. 37-69.

25. M akhoul RG, Fields CF, C assano AD. N itric oxide and the vascular surgeon. J Vasc Surg. 1999;30:569-72.

26. H owe LM, Boothe D M, Slater M R, Boothe HW, W ilkie S. $\mathrm{N}$ itric oxide generation in a rat model of acute portal hypertension. Am J V asc Res. 2000;61(10):1173-7.

27. Pereira M LL, Scheidt T C, Simoes M J, M osquette R, Gomes $P O . O$ xigenoterapia hiperbárica em lesões actínicas de colo de ratos: aspectos morfológicos e morfométricos. Acta Cir Bras. 2004;19:658-63.
28. $H$ artleb $M, M$ ichielsen PP, D ziurkowska-M arek $A$. T he role of nitric oxide in portal hypertensive systemic and portal vascular pathology. Acta G astroenterol Belg. 1997;60:222-32.

29. M oncada S, H igss EA. Endogenous nitric oxide: physiology, pathology and clinical relevance. Eur J Clin Invest. 1991;21: 361-74.

30. M oncadaS, Palmer RM J, H igssEA. N itric oxide: physiology, pathology and pharmacology. Pharmacol Rev. 1991;43: 109-42.

31. M azariegos GV, O 'T ooleK, M ielesLA, D vorchik I, M eza M, Briassoulis $G$, et al H yperbaric oxygen therapy for hepatic artery thrombosis after liver transplantation in children. Liver Transplant Surg. 1999;5:429-36.

\section{Correspondência:}

Ricardo Costa-Val

Rua Pium-í, 957/202, Bairro Sion

CEP 30310-080 - Belo H orizonte, M G

Tel.: (31) 9972.6066

E-mail: costaval@mkm.com.br 